\title{
Photonische Quasikristalle
}

\section{Laue-Beugung \\ auf dem Schreibtisch}

\author{
Alexandra Ledermann I Georg von Freymann I Martin Wegener
}

\section{Quasikristalle sind weder kristallin noch amorph. Sie be- stechen durch ihre Ästhetik und überraschende physikalische Eigenschaften. Inzwischen sind sogar dreidimensionale Quasikristalle nanotechnologisch herstellbar, die sichtbares Licht beugen. Ihre Laue-Beugungsbilder lassen sich mit einem Laserpointer studieren.}

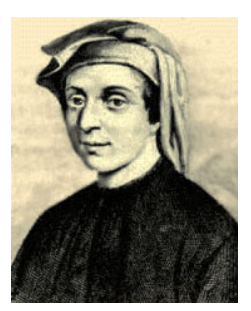

Leonardo da Pisa (ca. 1180-1240), Fibonacci genannt. für das Röntgenbeugungsbild des Kristalls. ristalle wie Kochsalz, Silizium oder Quarz bestehen aus ner immer gleichen Einheitszelle. Dieses Konstruktionsprinzip führt mathematisch zwingend dazu, dass nur bestimmte Symmetrien möglich sind. Insbesondere muss der Kristall bei Drehung um den $n$-ten Teil von $2 \pi$ in sich selbst übergehen, wobei die natürliche Zahl $n$ ausschließlich die Werte $n=1,2,3,4$ oder 6 annehmen kann (siehe „Zähligkeit von Drehachsen in Kristallen“, S. 303). Das Gleiche gilt

Im Jahr 1984 gab es deshalb eine faustdicke Überraschung für Physiker, Chemiker und Kristallographen: Dan Shechtman stellte eine sehr rasch verfestigte AluminiumMangan-Legierung vor, die der Physiker an der amerikanischen Johns Hopkins University untersucht hatte, und deren Röntgenbeugungsbilder $n=10$ Symmetrieachsen aufwiesen [1]. Ihre atomare Struktur konnte demnach nicht kristallin sein. Sie konnte aber auch nicht ungeordnet wie etwa ein Glas sein, denn sonst hätte sie kein derart symmetrisches Röntgenbeugungsbild gezeigt. Shechtman nannte die Struktur deshalb Quasikristall. Bei einer solchen Struktur befinden sich die Atome lokal in einer regelmäßigen Anordnung. Auf globaler Skala ist sie jedoch aperiodisch - jede Zelle ist von einem jeweils anderen Muster umgeben.

Atomare Strukturen beugen allerdings nur kurzwellige Röntgenstrahlung. Im Jahr 2006 gelang es uns in einer internationalen Kooperation zum ersten Mal mit nanotechnischen Methoden, dreidimensionale Quasikristalle mit so großen „künstlichen Atomen“ zu erzeugen, dass sie sichtbares Licht beugen. Durchleuchtet man zum Beispiel die fünfzählige Struktur in Abbildung 1 mit einem Laserpointer, dann erhält man entsprechende Laue-Beugungsbilder (Abbildung 2). Das Herstellungsverfahren haben wir gemein- sam mit kanadischen Kollegen von der Universität von Toronto entwickelt.

\section{Konstruktionsprinzipien}

Bei genauerer Betrachtung sind solche quasikristallinen Strukturen nicht ganz neu - zumindest nicht in ihrer einund zweidimensionalen Form. Der italienische Mathematiker Leonardo da Pisa (ca. 1180-1240), genannt Fibonacci, führte 1202 die nach ihm benannte Zahlenfolge $a_{m+1}=a_{m}$ $+a_{m-1}\left(\right.$ mit $a_{1}=0, a_{2}=1$ und Start bei $m=2$ ) ein.

In Analogie zur Fibonacci-Folge erhält man den eindimensionalen Quasikristall $Q_{m+1}$ des Iterationsschritts $m+1$ durch die Vorschrift $Q_{m+1}=Q_{m} \oplus Q_{m-1}$. Beginnt man mit den zwei unterschiedlichen Zellen $Q_{1}=A$ und $Q_{2}=B$, folgen die Sequenzen $Q_{3}=B A, Q_{4}=B A B, Q_{5}=B A B B A, Q_{6}=$ $B A B B A B A B, Q_{7}=B A B B A B A B B A B B A$ etc. (Abbildung 3 links). Stellt man die Zellen $A$ und $B$ durch zwei dielektrische Schichten mit Dicken im Bereich der Wellenlänge des Lichts dar, so ist die resultierende Schichtabfolge bereits ein eindimensionaler photonischer Quasikristall. Er kann durch Aufdampfen leicht hergestellt werden und weist interessante Eigenschaften bei der Ausbreitung von Lichtwellen auf [2].

Zweidimensionale Quasikristalle zieren als Muster schon den Darb-i-Imam-Schrein aus Isfahan (Iran), eine Begräbnisstätte aus dem Jahr 1453 [3]. Seine Wandfläche wird von drei unterschiedlich geformten Zellen parkettiert, die Girih-Kacheln heißen. Das Wort stammt aus dem Persischen und bedeutet „Knoten“. Diese Kacheln sind zu einer lückenlosen, aber wiederum aperiodischen Struktur mit lokal 10-zähliger Drehachse angeordnet. Ähnliche Flächen-Parkettierungen stellte Johannes Kepler (1571-1630) im zweiten Band seiner Harmonices Mundi libri vor, der 1619 erschien. Der englische Mathematiker Roger Penrose führte 1974 schließlich das heute nach ihm benannte PenroseParkett ein. Es gehört zu einer übergeordneten mathematischen Theorie für zweidimensionale Strukturen, aufgebaut aus zwei oder mehr unterschiedlichen Zellen [4] (Abbildung 3 rechts).

Normale Kristalle sind aber weder eindimensional noch flächig, sondern dreidimensionale, räumliche Strukturen. Kann man hier ein ähnlich klares Konstruktionsprinzip wie in einer oder zwei Dimensionen angeben? Die Antwort lau- 
tet ja. Dreidimensionale Quasikristalle können als schräge Projektion eines sechsdimensionalen Kristalls auf unsere dreidimensionale Welt aufgefasst werden [5,6]. Wir wollen diese entscheidende Aussage am Beispiel eines eindimensionalen Quasikristalls erläutern, der durch die Projektion aus zwei Dimensionen entsteht. Das Beispiel lässt sich dann recht leicht auf beliebige Dimensionen verallgemeinern: Generell hat der projizierte Kristall immer eine doppelt so hohe Dimension wie der Quasikristall.

Abbildung 4 zeigt ein einfaches Quadratgitter der Periode $a$, das um den Winkel $\alpha$ relativ zur $x$-Achse gedreht ist. Diese stellt den realen eindimensionalen Raum dar. Projiziert man nun alle Gitterpunkte auf die $x$-Achse, so ergibt sich eine Menge dicht liegender Punkte. Allgemein bilden sie weder einen eindimensionalen Kristall noch einen Quasikristall. Um diesen zu bekommen, brauchen wir ein Auswahlkriterium für die Projektion. Wir erhalten es, indem wir die Lagen der ursprünglichen Gitterpunkte bezüglich der $y$-Achse mit einbeziehen. Die $y$-Achse bildet den fiktiven oder internen Raum. Definieren wir hier ein „Einzugsgebiet" der Breite $\Delta$, so erhalten wir eine Teilmenge von Punkten. Diese liegt in dem Bereich, der in Abbildung 4 rot markiert ist.

Diese Teilmenge projizieren wir nun auf die $x$-Achse. Wählen wir beispielsweise einen Winkel von $\alpha=\pi / 4$, so ergibt sich eine Menge äquidistanter Punkte auf der $x$-Achse, also ein eindimensionaler Kristall, aber kein Quasikristall. Allgemein darf der Tangens des Winkels $\alpha$ keine rationale Zahl der Form $\tan \alpha=n / m$ sein, sonst schneidet die $x$-Achse nämlich periodisch ursprüngliche Gitterpunkte. Wählen wir hingegen beispielsweise den Kehrwert des Goldenen Schnitts

$(1+\sqrt{5}) / 2 \approx 1,6180$

also die irrationale Zahl

$\tan \alpha=2 /(1+\sqrt{5})$

so erhalten wir interessanterweise genau wieder unseren eindimensionalen Fibonacci-Quasikristall (Abbildung 3).

\section{Dreidimensionale photonische Quasikristalle}

Dieses Prozedere lässt sich nun relativ einfach von einer auf drei Dimensionen verallgemeinern: Aus dem Quadratgitter wird hierbei ein sechsdimensionales kubisches Gitter, aus dem Winkel $\alpha$ eine Drehung im Raum und aus der Breite $\Delta$ die Wigner-Seitz-Zelle des ursprünglichen Gitters, die als „Einzugsgebiet“ fungiert. Als Wigner-Seitz-Zelle bezeichnen Festkörperphysiker die primitive Elementarzelle mit dem kleinstmöglichen Volumen. Eine primitive Elementarzelle enthält nur einen Gitterpunkt in ihrem Zentrum. Drei der sechs Dimensionen spannen somit den realen Raum auf, die anderen drei den internen Raum.

Die Projektion eines Kristalls aus höheren Dimensionen wurde lange Zeit lediglich als elegante Möglichkeit gese-
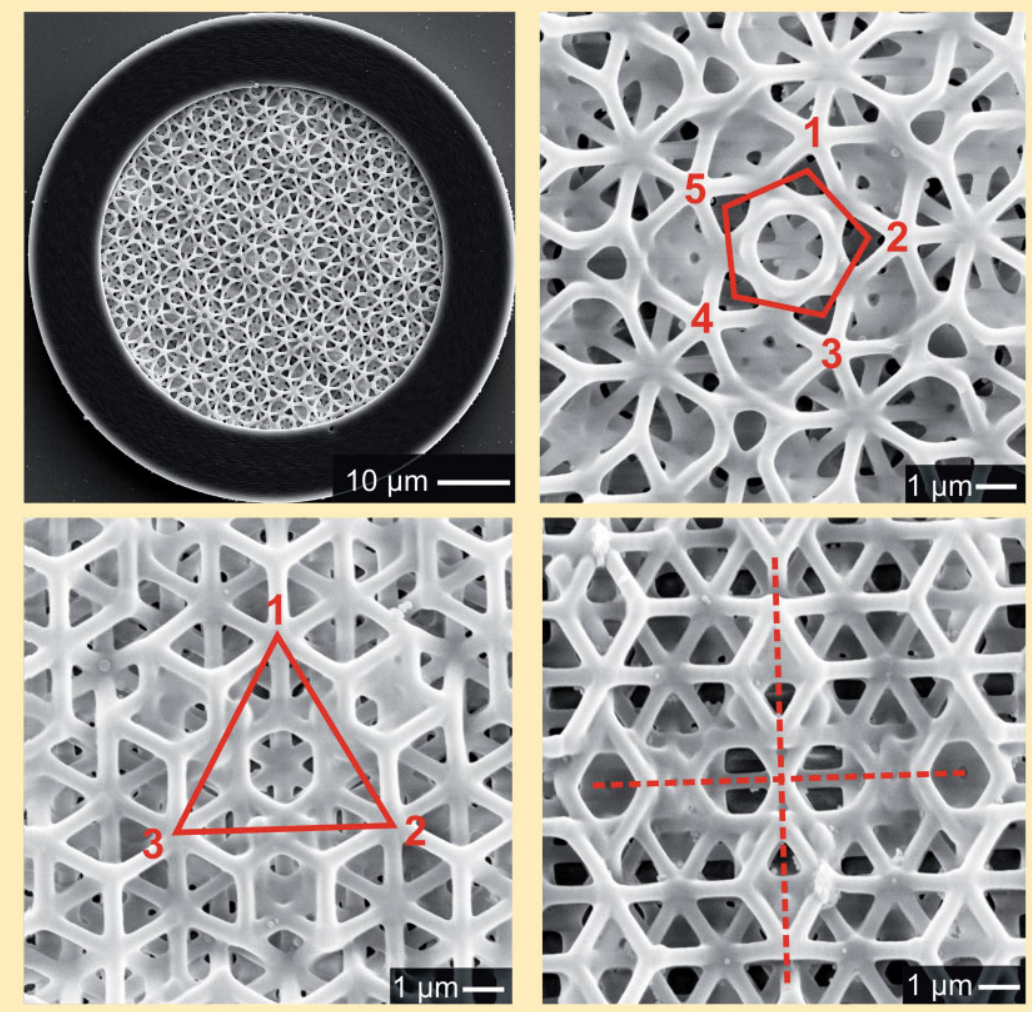

Abb. 1 Elektronenmikroskopische Aufnahmen eines dreidimensionalen photonischen Quasikristalls, der eine ikosaedrische Symmetrie aufweist [8]. Die verschiedenen Bilder entsprechen unterschiedlichen Orientierungen des gleichen Quasikristalls im Raum. Links oben: Die dicke Zylinderwand (in Aufsicht) stabilisiert die Struktur mechanisch.

hen, um reale Quasikristalle mathematisch darzustellen. Heute kann man diese Projektion jedoch auch direkt zur Herstellung von dreidimensionalen photonischen Quasikristallen verwenden $[7,8,9]$.
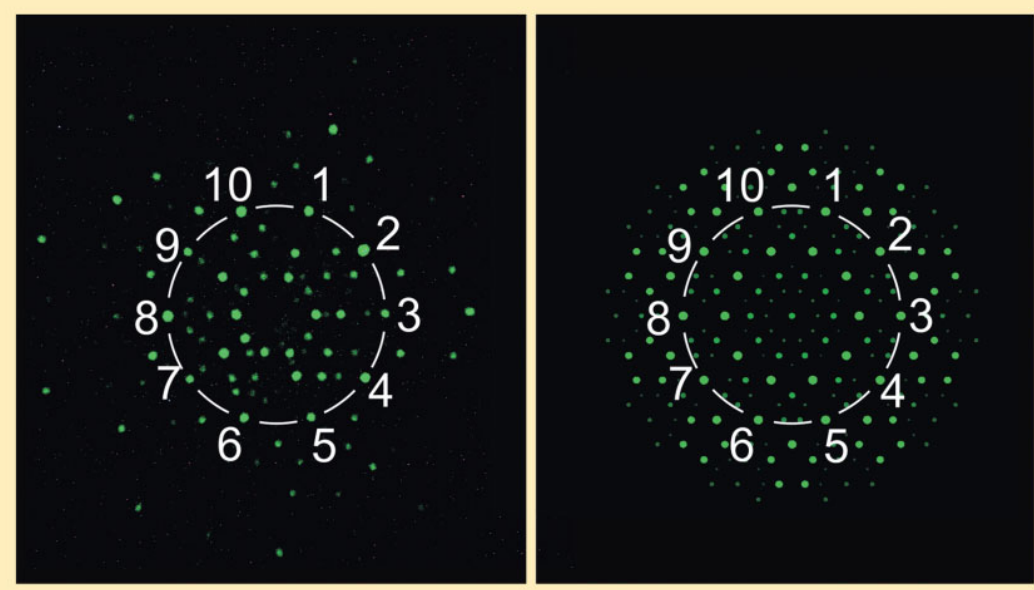

Abb. 2 Laue-Beugungsbilder des photonischen Quasikristalls aus Abbildung 1. Das linke Bild wurde mit einem grünen Laserpointer entlang einer fünfzähligen Drehachse aufgenommen. Es zeigt eine zehnzählige Drehachse, genauso wie das theoretisch berechnete, erwartete Beugungsbild (rechts) [8]. 


\section{ABB. 3 EIN- UND ZWEIDIMENSIONALER QUASIKRISTALL}
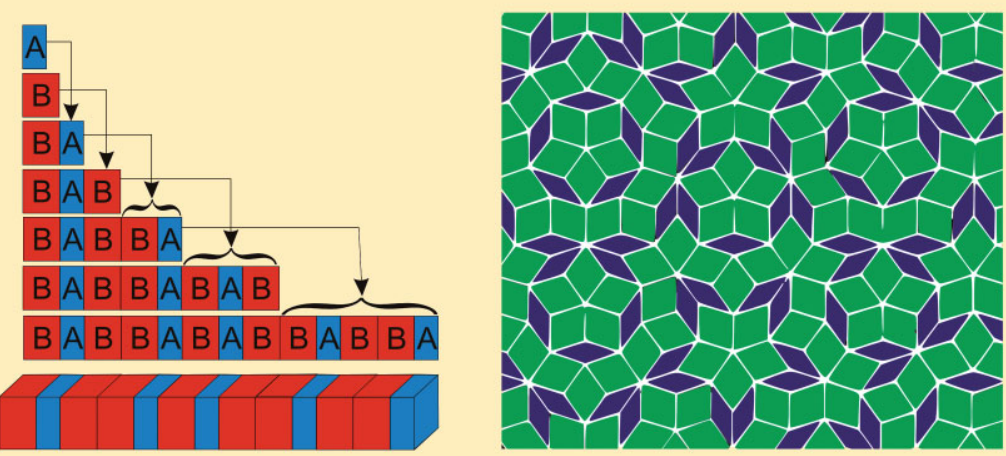

Konstruktionsprinzipien für ein- und zweidimensionale Quasikristalle.

Links: Die Fibonacci-Konstruktion ordnet zwei unterschiedliche Zellen A und B zu einem eindimensionalen Quasikristall an. Rechts: Die Parkettierung der Fläche mit zwei Zellen (oder Kacheln) A und B ergibt einen zweidimensionalen Quasikristall (Penrose-Parkett).

Bei diesem Verfahren wird zuerst die Projektion eines sechsdimensionalen Kristalls im Computer durchgerechnet. Die daraus resultierenden Gitterpunkte des dreidimensionalen Quasikristalls werden dann mit einem laserbasierten Lithographieverfahren in eine reale Polymerstruktur übersetzt. Dazu schleudern wir zunächst eine dicke Schicht eines lichtempfindlichen Lacks auf einen Glasträger auf. Dann fokussieren wir Lichtimpulse aus einem Femtosekundenlaser in das Volumen des Lacks. Nur in einem kleinen räum-

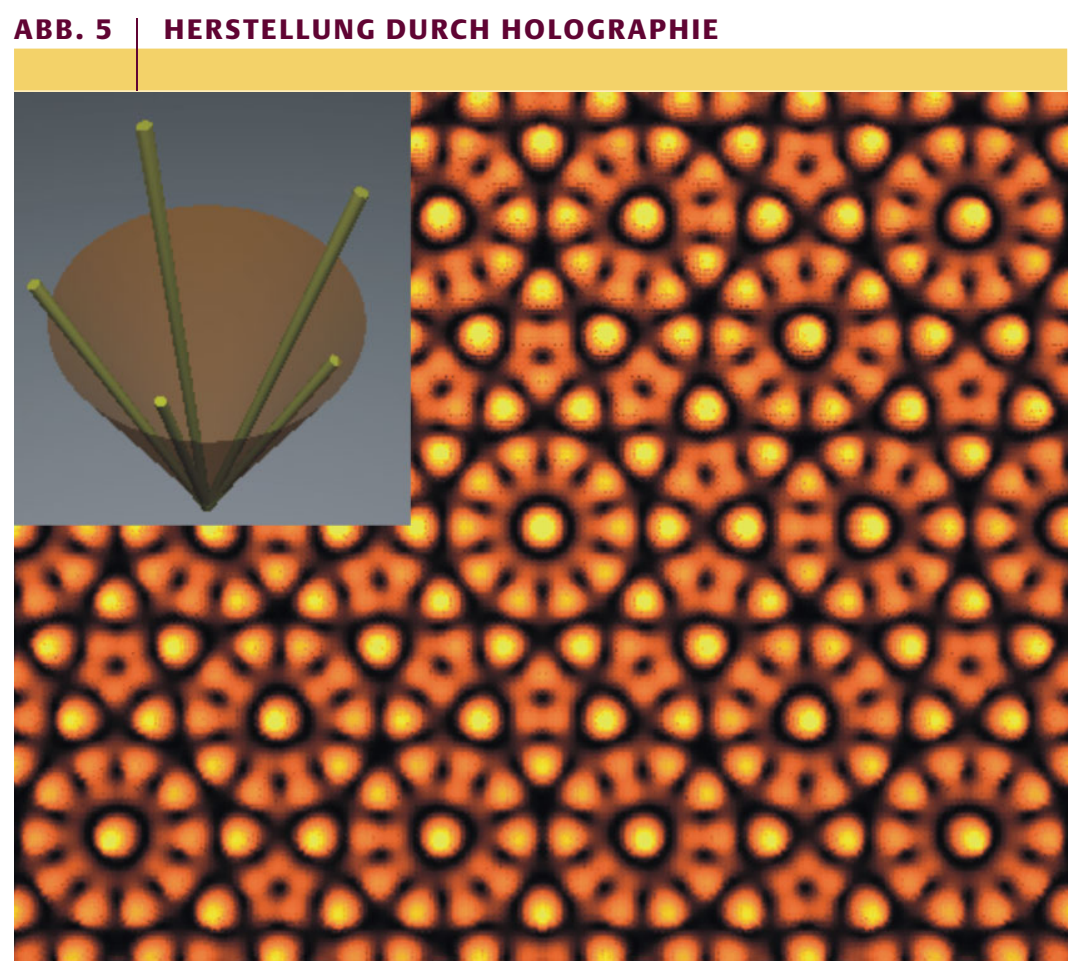

Links oben: Fünf monochromatische, kohärente (unfokussierte) Laserstrahlen überlagern sich in einem gewissen Bereich. Das dort entstehende Intensitätsmuster besitzt eine zehnzählige lokale Symmetrie (großes Bild) und eignet sich zum Belichten entsprechender quasikristalliner Strukturen.

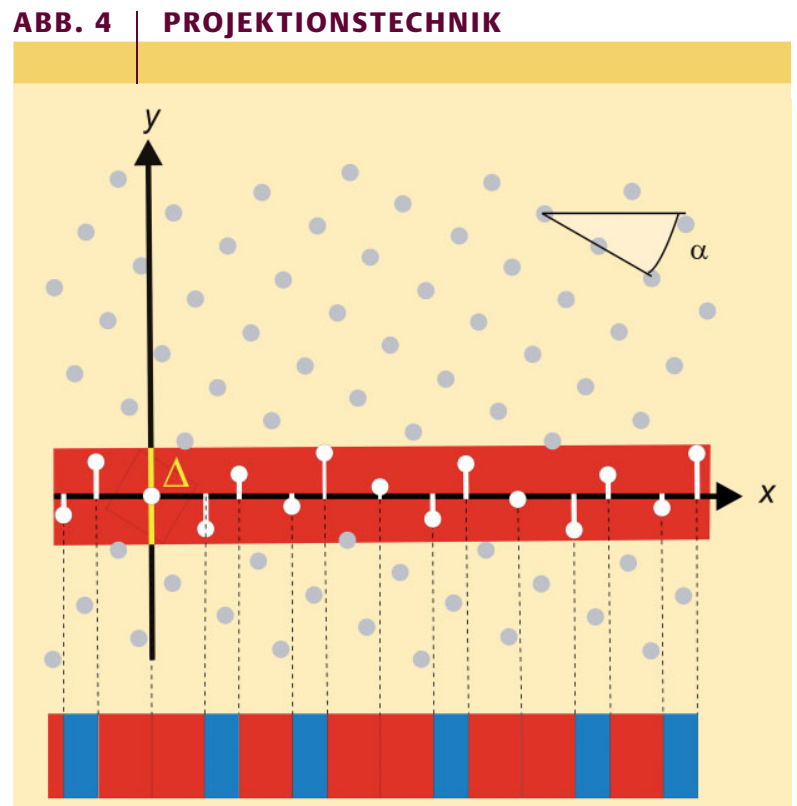

Bei der „Abschneide- und Projektionstechnik“ wird ein zweidimensionales quadratisches Kristallgitter um einen bestimmten Winkel $\alpha$ gedreht und auf den realen Raum auf der $x$-Achse projiziert. Bei der hier gezeigten Drehung mit tan $\alpha=2 /(1+\sqrt{5})$ entsteht ein eindimensionaler FibonacciQuasikristall.

lichen Bereich im Fokus wird der Photolack durch Zweiphotonenabsorption ausreichend stark belichtet (siehe auch Physik in unserer Zeit 2005, 36 (6), 279). Die Zweiphotonenabsorption ist unbedingt notwendig, weil sie das gaußförmige Laserprofil im Fokus im Wesentlichen quadriert. So erreicht sie eine deutlich stärkere räumliche Konzentration der Belichtung als die Einphotonenabsorption.

Wir können so nahezu beliebige dreidimensionale Strukturen erzeugen. Dazu verfahren wir auf einem piezoelektrischen Tisch, der in allen drei Dimensionen per Computer steuerbar ist, den Laserfokus nanometergenau relativ zum Lack. Die hinreichend belichteten Bereiche polymerisieren - also vernetzen - im anschließenden Backprozess. Sie werden deshalb im Entwicklerbad nicht herausgewaschen. Das Resultat ist eine dreidimensionale Polymerstruktur, die ein digitalisiertes Abbild des Belichtungsprozesses darstellt [8].

Abbildung 1 zeigt verschiedene elektronenmikroskopische Aufnahmen eines so hergestellten ikosaedrischen Quasikristalls, hier ein Beispiel mit einer fünfzähligen Drehachse. Man kann deutlich erkennen, dass die gedachten Gitterpunkte durch kleine dünne Stäbe mit einer Länge von zwei Mikrometern verbunden sind. Das ist bei photonischen Quasikristallen unbedingt notwendig, denn schließlich können die Gitterpunkte nicht in der Luft schweben.

\section{Laue-Beugungsbilder im Sichtbaren}

Es ist klar, dass eine einfallende elektromagnetische Welle nur von der dielektrischen Struktur gebeugt wird, wenn ih- 
re Wellenlänge im Bereich des Abstandes benachbarter Gitterpunkte oder darunter liegt. Im Gegensatz zu Shechtmans Quasikristallen, bei denen die atomaren Gitterpunkte nur wenige Zehntel eines Nanometers voneinander entfernt sind, bekommen wir bei den photonischen Quasikristallen ein Beugungsbild im sichtbaren Spektrum. Das in Abbildung 1 gezeigte Beispiel einer Struktur mit ikosaedrischer Symmetrie (Ikosaeder: Zwanzigflächner) kann mit einem grünen Laserpointer mit $532 \mathrm{~nm}$ Wellenlänge aufgenommen werden. Anstatt einer aufwendigen Röntgenapparatur erhalten wir so ein Experiment, das leicht auf jeden Schreibtisch passt.

Abbildung 2 zeigt, wie schon erwähnt, das gemessene Laue-Beugungsbild. Die rechte Seite von Abbildung 2 stellt das theoretisch erwartete Beugungsbild daneben [8]. Die fünfzählige Drehachse im Ortsraum der Struktur führt zu einer zehnzähligen Drehachse im Beugungsbild. Das ist das optische Analogon [8] zu den ikosaedrischen AluminiumMangan-Legierungen von Shechtman [1].

\section{Quasikristalle durch Holographie}

Den Prozess der Beugung kann man im Prinzip auch umkehren. Dabei synthetisiert man den photonischen Quasikristall holographisch, indem man mehrere Laserstrahlen entlang der Richtungen der Beugungsmaxima einstrahlt. Abbildung 5 zeigt das Verfahren. Dabei werden fünf monochromatische kohärente Laserstrahlen der Frequenz $\omega$ in einer Ebene überlagert. Die Strahlrichtungen sind wie gezeigt mit gleichmäßigen Abständen auf einem gedachten Kegelmantel angeordnet. Die $z$-Achse des Koordinatensystems ist so gewählt, dass sie mit der zentralen Achse des Kegels zusammenfällt. In der Überlagerungsebene $(z=0)$ entsteht so ein Stehwellenmuster auf der $x y$-Ebene (großes Bild). Es ergibt sich aus der räumlichen Variation der Lichtintensität

$I(\boldsymbol{r})=I(x, y, z)$

also aus dem Zeitmittel des Betragsquadrats der Summe aus fünf ebenen Wellen

$I(\boldsymbol{r}) \propto\left\langle\left|\sum_{m=1}^{5} \cos \left(\boldsymbol{k}_{m} \boldsymbol{r}-\omega t\right)\right|^{2}\right\rangle$

mit den Lichtwellenvektoren

$\boldsymbol{k}_{m} \propto\left(\cos \left(\frac{m}{5} \cdot 2 \pi\right), \sin \left(\frac{m}{5} \cdot 2 \pi\right),-1\right)$.

Die Intensitätsverteilung $I(\boldsymbol{r})$ entspricht einem zweidimensionalen Quasikristall mit lokal fünfzähliger Drehachse [10]. Benutzt man sie wie schon vorgestellt zur Belichtung von einem Photolack, dann kann man auch so eine quasikristalline Struktur herstellen.

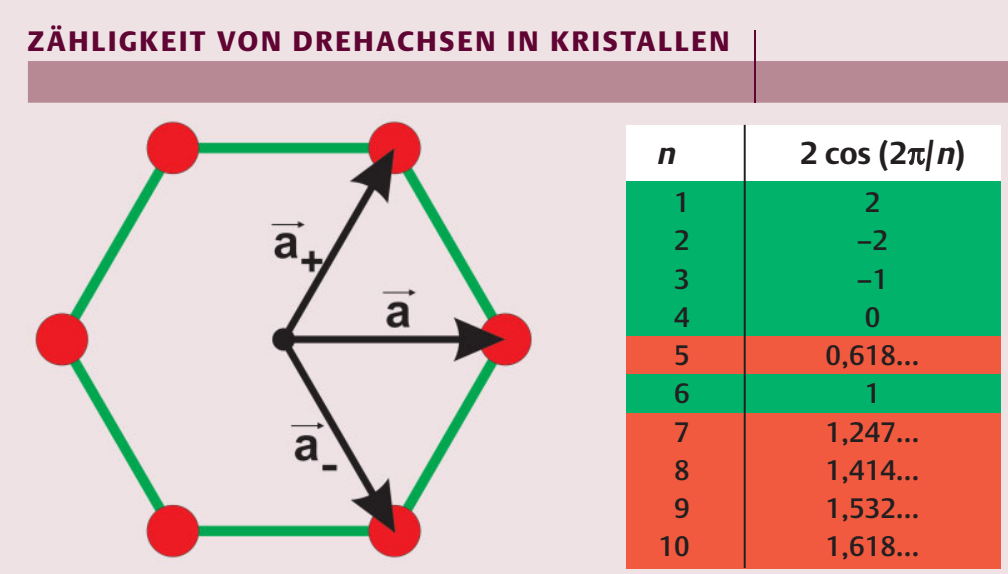

Links: Zähligkeit am Beispiel eines hexagonalen Gitters, a sind Gittervektoren. Rechts: Tabelle für Drehungen.
Der abgebildete Gittervektor

$a=a(1,0,0)$

führt von einem Gitterpunkt des Kristalls zu einem unmittelbar benachbarten Gitterpunkt. Die Einser und Nuller bezeichnen die Komponenten des Vektors in einem kartesischen Koordinatensystem in Einheiten von $a$. $(1,0,0)$ bedeutet im abgebildeten Beispiel also „nach rechts zeigend“. Die Zähligkeit der Gittersymmetrie ist so definiert, dass das Gitter nach einer passenden Drehung um den Winkel $\pm 2 \pi / n$ um die $z$-Achse in sich selbst übergehen muss. Folglich müssen auch die entsprechend rechts- oder linksherum gedrehten Vektoren

$a_{ \pm}=a(\cos (2 \pi / n), \pm \sin (2 \pi / n), 0)$
Gittervektoren sein (Abbildung). Somit ist $\boldsymbol{a}_{+}+\boldsymbol{a}_{-}$ebenfalls ein Gittervektor. $\mathrm{Er}$ ist allerdings kein unabhängiger Vektor, denn es muss

$a_{+}+a_{-}=m a$

mit ganzzahligem $m$ gelten. Aus der ersten Komponente dieses Vektors folgt unmittelbar die Beziehung

$2 \cos (2 \pi / n)=m$.

Die Tabelle (Abbildung rechts) zeigt, dass der Ausdruck $2 \cos (2 \pi / n)$ nur für die Zahlen $n=1,2,3,4$ und 6 ganzzahlige Werte $m$ annimmt. Eine fünfoder zehnzählige Drehachse kann es in Kristallen also nicht geben - in Quasikristallen hingegen schon.
Damit wird klar, dass Laue-Beugung und Holographie ein ähnliches Paar bilden wie Fourier-Analyse und FourierSynthese. Auch dreidimensionale Quasikristalle lassen sich durch Interferenz von einigen Laserstrahlen holographisch synthetisieren. Durch sechs, sieben oder zehn Laserstrahlen beispielsweise kann so im Ortsraum eine lokal fünfzählige Drehachse entstehen.

Das Studium der photonischen Quasikristalle ist auch deshalb interessant, weil sich das Licht in ihnen komplexer verhält als die Röntgenstrahlung bei den atomaren Strukturen. Das Licht „spürt“ nämlich eine starke räumliche Modulation der optischen Brechzahl in der photonischen Struktur. Das gilt besonders, wenn der photonische Quasikristall aus Silizium aufgebaut ist. Bei diesen Objekten birgt die Physik der Wellenausbreitung noch spannende Rätsel. Diese gilt es in den kommenden Jahren zu lösen. 


\section{Zusammenfassung}

Quasikristalle aus atomaren Strukturen, die Röntgenlicht beugen, sind seit rund einem Vierteljahrhundert bekannt. Seit 2006 sind dreidimensionale Quasikristalle mit größeren Strukturen nanotechnologisch herstellbar, die auf sichtbares Licht abgestimmt sind. Diese photonischen Quasikristalle erlauben zum Beispiel mit einem Laserpointer eine einfache Visualisierung der ungewöhnlichen Laue-Beugungsbilder. Im Gegensatz zum Röntgenbereich „spürt“ das Licht beim Durchgang durch die Struktur eine starke räumliche Modulation der optischen Brechzahl. Das wirft neue Fragen zur Physik der Wellenausbreitung in photonischen Quasikristallen auf.

\section{Stichworte}

Photonische Quasikristalle, Laue-Beugung, ikosaedrische Symmetrie.

\section{Literatur}

[1] D. Shechtman et al., Phys. Rev. Lett. 1984, 53, 1951.

[2] L. Dal Negro et al., Phys. Rev. Lett. 2003, 90, 055501.

[3] P. J. Lu und P. J. Steinhardt, Science 2007, 315, 1106.

[4] B. Grünbaum und G. C. Shepard, Tilings and Patterns, W.H. Freeman and Company, New York, 1987.

[5] P. J. Steinhardt und S. Ostlund, The Physics of Quasicrystals, World Scientific, Singapore, 1987.

[6] C. Janot, Quasicrystals - A Primer, Clarendon, Oxford, 1992.

[7] W. Man et al., Nature 2005, 436, 993.

[8] A. Ledermann et al., Nature Mater. 2006, 5, 942.

[9] C. T. Chan, Nature Photonics 2007, 1, 91

[10] B. Freedman et al., Nature 2006, 440, 1166.

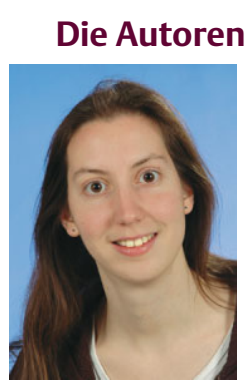

Alexandra Ledermann studierte Physik und promoviert in der Arbeitsgruppe von Martin Wegener und Georg von Freymann am Institut für Nanotechnologie des Forschungszentrums Karlsruhe.

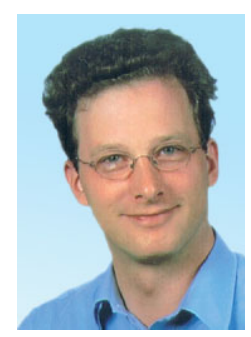

Georg von Freymann ist Leiter einer Emmy-NoetherNachwuchsgruppe der Deutschen Forschungsgemeinschaft (DFG) am Institut für Nanotechnologie des Forschungszentrums Karlsruhe und am Institut für Angewandte Physik der Universität Karlsruhe $(T H)$.

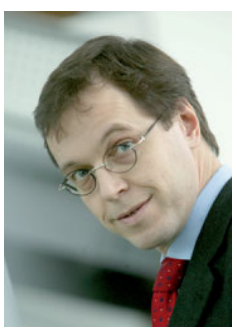

Martin Wegener ist Ordinarius am Institut für Angewandte Physik der Universität Karlsruhe (TH), Arbeitsgruppenleiter am Institut für Nanotechnologie des Forschungszentrums Karlsruhe in der Helmholtz-Gemeinschaft und Koordinator des Karlsruher DFG-Forschungszentrums für Funktionelle Nanostrukturen (CFN).

Anschrift

Alexandra Ledermann, Dr. Georg von Freymann, Prof. Dr. Martin Wegener, DFG-Center for Functional Nanostructures, Universität Karlsruhe (TH), 76128 Karlsruhe.

martin.wegener@physik.uni-karlsruhe.de. 